\title{
Five-Year Outcomes of a Randomized Trial of Treatments for Varicose Veins
}

The authors' affiliations are listed in the Appendix. Address reprint requests to Dr. Brittenden at the Institute of Cardiovascular Research, University of Glasgow, Glasgow G81 4HX, United Kingdom, or at julie.brittenden@glasgow.ac.uk.

N Engl J Med 2019;381:912-22. DOI: 10.1056/NEJMoal805186 Copyright () 2019 Massachusetts Medical Society.

\author{
Julie Brittenden, M.D., David Cooper, Ph.D., Maria Dimitrova, M.Sc., \\ Graham Scotland, Ph.D., Seonaidh C. Cotton, Ph.D., Andrew Elders, M.Sc., \\ Graeme MacLennan, M.Sc., Craig R. Ramsay, Ph.D., John Norrie, M.Sc., \\ Jennifer M. Burr, M.D., Bruce Campbell, M.B., B.S., Paul Bachoo, M.B., Ch.B., \\ Ian Chetter, M.B., Ch.B., M.D., Michael Gough, M.B., Ch.B., Jonothan Earnshaw, D.M., \\ Tim Lees, M.B., Ch.B., M.D., Julian Scott, M.B., Ch.B., M.D., Sara A. Baker, M.Sc., \\ Emma Tassie, M.Sc., Jill Francis, Ph.D., and Marion K. Campbell, Ph.D.
}

\section{A B S TRACT}

\section{BACKGROUND}

Endovenous laser ablation and ultrasound-guided foam sclerotherapy are recommended alternatives to surgery for the treatment of primary varicose veins, but their long-term comparative effectiveness remains uncertain.

\section{METHODS}

In a randomized, controlled trial involving 798 participants with primary varicose veins at 11 centers in the United Kingdom, we compared the outcomes of laser ablation, foam sclerotherapy, and surgery. Primary outcomes at 5 years were disease-specific quality of life and generic quality of life, as well as cost-effectiveness based on models of expected costs and quality-adjusted life-years (QALYs) gained that used data on participants' treatment costs and scores on the EuroQol EQ-5D questionnaire.

\section{RESULTS}

Quality-of-life questionnaires were completed by 595 (75\%) of the 798 trial participants. After adjustment for baseline scores and other covariates, scores on the Aberdeen Varicose Vein Questionnaire (on which scores range from 0 to 100, with lower scores indicating a better quality of life) were lower among patients who underwent laser ablation or surgery than among those who underwent foam sclerotherapy (effect size [adjusted differences between groups] for laser ablation vs. foam sclerotherapy, -2.86 ; $95 \%$ confidence interval [CI], -4.49 to $-1.22 ; \mathrm{P}<0.001$; and for surgery vs. foam sclerotherapy, $-2.60 ; 95 \% \mathrm{CI},-3.99$ to -1.22 ; $\mathrm{P}<0.001)$. Generic quality-oflife measures did not differ among treatment groups. At a threshold willingness-topay ratio of $£ 20,000$ ( $\$ 28,433$ in U.S. dollars) per QALY, $77.2 \%$ of the cost-effectiveness model iterations favored laser ablation. In a two-way comparison between foam sclerotherapy and surgery, $54.5 \%$ of the model iterations favored surgery.

\section{CONCLUSIONS}

In a randomized trial of treatments for varicose veins, disease-specific quality of life 5 years after treatment was better after laser ablation or surgery than after foam sclerotherapy. The majority of the probabilistic cost-effectiveness model iterations favored laser ablation at a willingness-to-pay ratio of $£ 20,000(\$ 28,433)$ per QALY. (Funded by the National Institute for Health Research; CLASS Current Controlled Trials number, ISRCTN51995477.) 
M

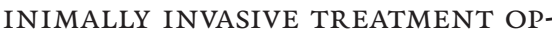
tions such as endovenous laser ablation therapy and ultrasound-guided foam sclerotherapy are now recommended alternatives to surgery for the treatment of varicose veins. ${ }^{1-9}$ Medium-term follow-up studies suggest that laser ablation, foam sclerotherapy, and surgery result in equivalent recurrence rates and quality of life at 2 years and 3 years after treatment. ${ }^{10-12}$ At 5 years, quality of life appears to be similar among those who underwent laser ablation and those who underwent surgery, ${ }^{10,13-15}$ but limited comparative data are available on persons who underwent foam sclerotherapy. ${ }^{16}$ There is also a paucity of data on clinical recurrence rates, which has led to uncertainty regarding the cost-effectiveness of these treatments.

We performed the Comparison of Laser, Surgery, and Foam Sclerotherapy (CLASS) trial, a large, multicenter, randomized, comparativeeffectiveness trial, to assess quality of life and cost-effectiveness associated with treatment for varicose veins at 6 months and 5 years after treatment. ${ }^{9,17,18}$ Here, we present the 5 -year primary outcomes of the CLASS trial.

\section{METHODS}

\section{STUDY OVERSIGHT}

This study was conducted according to the protocol, available with the full text of this article at NEJM.org. ${ }^{9}$ Approval of the trial for all sites was obtained from the Scotland A Research Ethics Committee and the Medicines and Healthcare Products Regulatory Authority. Aberdeen University was responsible for initiation, management, and financial oversight of the research. The trial was overseen by a trial steering committee and an independent data and safety monitoring committee. Data analysis was performed by statisticians at the Centre for Healthcare Randomized Trials, University of Aberdeen. The project management group (see the Supplementary Appendix, available at NEJM.org) assumes responsibility for the accuracy and completeness of the data and the fidelity of the trial to the protocol.

\section{PATIENTS}

Patients who were eligible for treatment of varicose veins were recruited at 11 vascular surgery departments in the United Kingdom from November 2008 through October 2012. Inclusion criteria were an age of 18 years or older, the presence of primary symptomatic varicose veins greater than $3 \mathrm{~mm}$ in diameter in one or both legs, and reflux of the great saphenous or small saphenous veins of more than 1 second as measured by duplex ultrasonography. In participants with varicose veins in both legs, the more severely affected leg was designated as the study leg. Exclusion criteria were current deep-vein thrombosis, acute superficial-vein thrombosis, and additional anatomical and participant factors (listed in Table S1 in the Supplementary Appendix). Written informed consent was obtained from all participants.

\section{RANDOMIZATION AND STUDY TREATMENT}

We compared foam sclerotherapy, laser ablation (with subsequent foam sclerotherapy for residual varicosities, if necessary), and surgery. Participants underwent randomization with the use of a computer-generated randomization system, with balanced assignment to all treatment options available at each investigating center within two strata - stratum A, which included eight hospitals offering all three treatment options; and stratum B, which included three hospitals offering treatment only with foam sclerotherapy and surgery. Randomization was performed with the use of a minimization algorithm that included center, age ( $<50$ years or $\geq 50$ years), sex, reflux in the great or the small saphenous veins, and varicose veins in one or both legs. Because of the nature of the treatment, participants and assessors were aware of the group assignments.

The laser ablation, foam sclerotherapy, and surgical treatment methods have been described previously (Table S2 in the Supplementary Appendix). ${ }^{9}$ Laser ablation was performed, with the patient under local anesthesia, in a treatment room in the case of $90.5 \%$ of legs treated and in an operating theater in the case of $9.5 \%$. Surgery was performed while the patient was under general anesthesia in an ambulatory care setting. In patients with varicose veins in both legs, both legs were treated with the same method when possible.

\section{FOLLOW-UP AND OUTCOME MEASURES}

Five years after treatment, all participants were invited to attend a 5-year follow-up clinic appointment. If they did not attend, they were mailed participant questionnaires followed by two mailed 
reminders. The medical records of all participants were reviewed by a trial nurse to determine the timing and nature of any treatment the participants received after the initial procedure.

The primary outcome measures at 5 years were participant-reported disease-specific quality of life measured with the Aberdeen Varicose Vein Questionnaire (AVVQ) $)^{19-22}$; participant-reported generic quality of life measured with the EuroQol EQ-5D (EQ-5D) questionnaire ${ }^{23}$ and the Medical Outcomes Study 36-Item Short-Form Health Survey (SF-36) ${ }^{24}$ (physical-component and mental-component scores); and 5-year modelbased cost-effectiveness calculated as cost per quality-adjusted life-year (QALY) gained. The AVVQ consists of 12 questions and a set of mannequin legs on which participants are asked to draw their veins. AVVQ scores range from 0 to 100 , with lower scores indicating a better quality of life. The EQ-5D has five dimensions, and scores range from -0.594 to 1.000 , with higher scores indicating a better quality of life. The SF-36 consists of eight domains and yields two summary scores (the physical component score and the mental component score), each of which ranges from 0 to 100 , with higher scores indicating greater well-being. Our previously constructed Markov model was updated with observed rates of recurrence and further treatment, and the cost and utility impact (i.e., the effect of recurrence and further treatment on the cost and effects outcomes) of these events were derived from the 5-year analysis of individual patient data. ${ }^{17,18}$ (Additional details on the Markov model and on all scales and scores included in this article are provided in Fig. S1 and Table S3 in the Supplementary Appendix.)

Secondary outcomes were quality of life measured with the EQ-5D visual-analogue scale (VAS; scores range from 0 to 100 , with higher scores indicating better health) and the SF-36 domain scores; trial-based incremental cost-effectiveness, assessed with the use of data on costs and QALYs for individual patients; clinical success as measured by the presence of varicose veins assessed by the participant and nurse using a VAS that ranged from 0 (no varicose veins) to 10 (the worst varicose veins the patient can imagine) and the Venous Clinical Severity Score (a score consisting of nine categories relating to symptoms or signs of venous disease and one cate- gory relating to the use of compression; scores range from 0 [no venous disease] to 30 [most severe venous disease]); additional procedures for treatment of varicose veins and the associated costs; and participant satisfaction and willingness to repeat and recommend the treatment. In addition, the effect of the treatments on truncal-vein ablation, as assessed by duplex ultrasonography, was determined in a subgroup of participants.

\section{STATISTICAL ANALYSIS}

We performed intention-to-treat analyses for the following prespecified comparisons: a comparison of foam sclerotherapy with surgery using data from participants in strata A and B, a comparison of laser ablation with surgery using data from patients in stratum A, and a comparison of laser ablation with foam sclerotherapy using data from patients in stratum A. The principal analyses of the trial were performed when all participants had completed a 5-year follow-up and were conducted according to a prespecified statistical plan (available with the protocol at NEJM.org), with the use of Stata software, version 14 (StataCorp). The study database was locked on August 15, 2017, before the analyses were performed.

To determine the treatment effect size, we compared the groups using general linear models with adjustments for the minimization covariates and, when possible, with adjustments for baseline scores on the AVVQ, EQ-5D, SF-36, and Venous Clinical Severity Score. Mixed models with a compound-symmetry covariance matrix and with the center fitted as a random effect were used to analyze the continuous outcomes. All the available data were used to estimate effect sizes and interactions, and any missing data were assumed to be missing at random. Details of available interval quality-of-life data are included in Table S4 in the Supplementary Appendix. Sensitivity analyses were performed to compare the demographic characteristics of patients who completed the 5-year follow-up with those of patients who did not complete the 5-year followup. ${ }^{25}$ As an additional sensitivity analysis, multiple imputation with chained equations was performed for the primary quality-of-life outcomes at 5 years. We analyzed truncal-vein ablation rates and treatment satisfaction using ordinal logistic regression. 
Details of the original power calculation were published previously.9 The initial planned sample size was 1015 patients but was later revised to 779 on the basis of results of a review by the data and safety monitoring committee. The objective was to have at least $90 \%$ power to detect a difference of 0.25 standard deviation in the AVVQ score for the comparison of foam sclerotherapy with surgery at 6 months; this difference was considered to be clinically significant. The sample size calculations accounted for an estimated 30\% loss to follow-up at 5 years. A statistical significance level of $5 \%$ was adjusted to account for multiple comparisons $(\mathrm{P}<0.0033)$ for primary outcomes. Confidence intervals have not been adjusted for multiplicity, and therefore inferences drawn from these intervals may not be reproducible.

Both the model-based and trial-based economic analyses included a three-way comparison of foam sclerotherapy, surgery, and laser ablation with participant data from stratum $\mathrm{A}$ and a two-way comparison (foam sclerotherapy vs. surgery) with data from Strata A and B. The statistical methods for the economic analyses are provided in Tables S5 and S6 and the text in the Supplementary Appendix.

\section{RESULTS}

\section{PATIENTS AND TREATMENT}

We recruited 798 participants, of whom 595 (75\%) completed the participant questionnaires at 5 years, including questionnaires assessing quality of life, presence of varicose veins, satisfaction with treatment, and further treatment they had received (Fig. 1). The baseline demographic and clinical data are shown in Table 1. A sensitivity analysis showed that participants who did not complete the 5-year follow-up questionnaires were younger than those who did complete the questionnaires (mean age, 44.5 years vs. 51.0 years; $\mathrm{P}<0.001$ ) (Table $\mathrm{S} 7$ in the Supplementary Appendix).

Data on 766 participants (96\%) were extracted from case notes. In total, 467 participants (59\%) attended a clinic visit at which a nurse assessed the secondary outcomes of Venous Clinical Severity Score and extent of varicose veins; 389 participants (49\%) underwent duplex ultrasonography, which was performed at seven centers.

\section{PRIMARY OUTCOMES}

Table 2 summarizes results for the four primary quality-of-life outcome measures. All groups showed improvement from baseline in quality of life. The AVVQ scores in the laser ablation and surgery groups were significantly lower (indicating better quality of life) than the scores in the foam sclerotherapy group (effect size for laser ablation vs. foam sclerotherapy, -2.86 ; $95 \%$ confidence interval $[\mathrm{CI}],-4.49$ to $-1.22 ; \mathrm{P}<0.001$; and effect size for surgery vs. foam sclerotherapy, $-2.60 ; 95 \%$ CI, -3.99 to -1.22 ; $\mathrm{P}<0.001$ ). A sensitivity analysis revealed no significant differences in the baseline or 6-month AVVQ scores between patients who did not complete the 5 -year follow-up and those who completed the 5-year follow-up. The other quality-of-life measures did not differ significantly among the treatment groups. Sensitivity analyses of the quality-of-life measures with the use of multiple imputation with chained equations revealed similar results. (Also see Tables S8 and S9 in the Supplementary Appendix.)

The updated Markov model results are shown in Table 3. In the three-way model-based costeffectiveness analysis, laser ablation cost $£ 460$ (\$654 in U.S. dollars) more than foam sclerotherapy and generated a nonsignificant gain in QALYs (0.113), for an incremental cost-effectiveness ratio of $£ 4,064(\$ 5,778)$ per QALY gained. Surgery cost $€ 289$ (\$411) more than laser ablation and generated marginally fewer QALYs; as a result, from a cost-effectiveness standpoint, laser ablation was deemed to be superior to surgery. At a threshold willingness-to-pay ratio of $£ 20,000$ $(\$ 28,433)$ per QALY, $77.2 \%$ of the probabilistic model iterations favored laser ablation (Fig. 2). Figure S2 in the Supplementary Appendix shows the uncertainty surrounding the joint incremental costs and effects for the three-way comparison.

In the two-way comparison of foam sclerotherapy with surgery, surgery cost an additional $£ 737(\$ 1,048)$ per patient, for a mean QALY gain of 0.042 , resulting in an incremental cost-effectiveness ratio of $€ 17,554 \quad(\$ 24,956)$ (Table 3 ). Figure S3 in the Supplementary Appendix shows the uncertainty surrounding the joint incremental costs and effects for the two-way comparison. At the willingness-to-pay threshold of $£ 20,000(\$ 28,433)$ per QALY, $54.5 \%$ of the model iterations favored surgery (Fig. S4 in the Supplementary Appendix). 


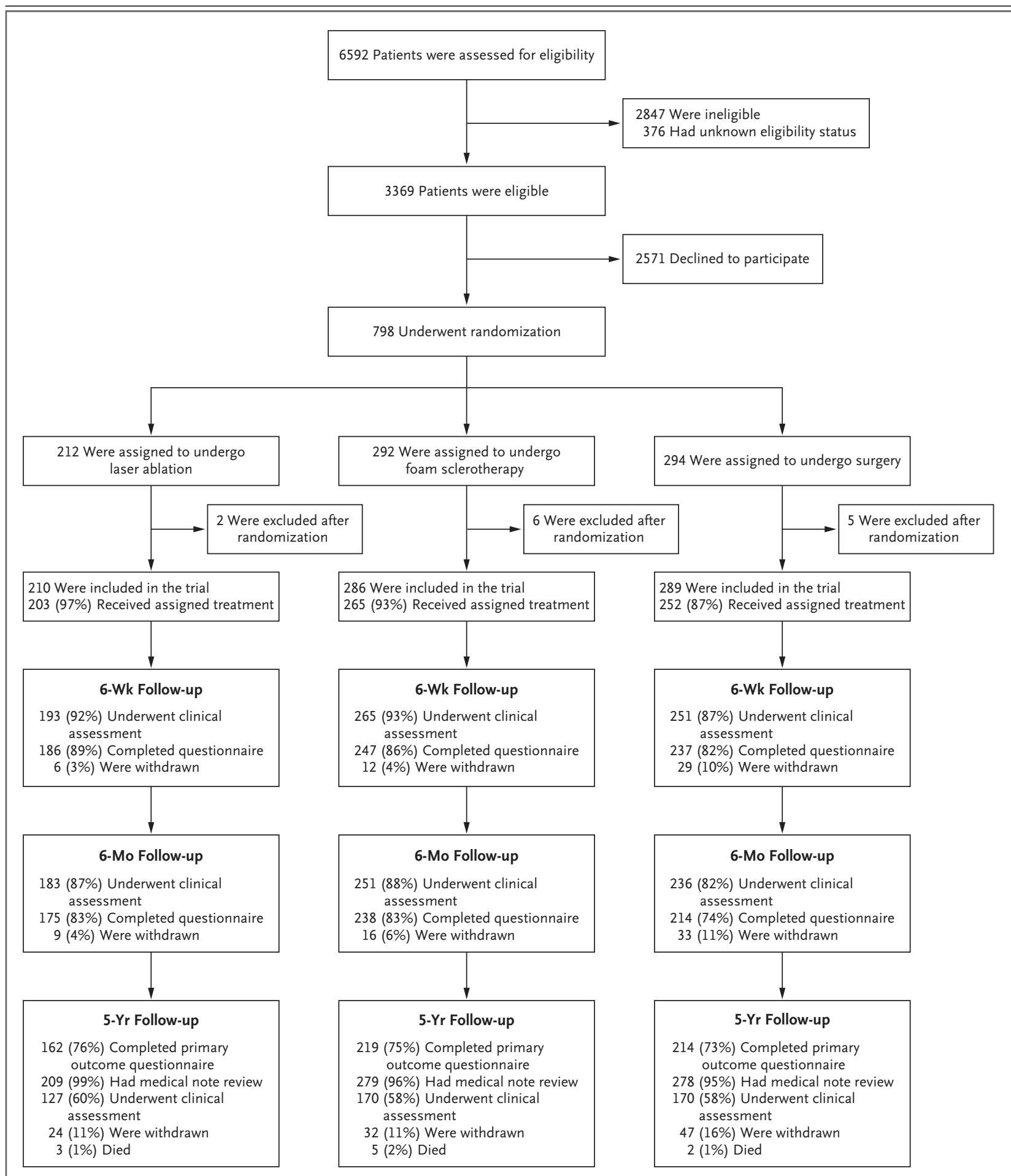

Figure 1. Screening, Randomization, and Follow-up.

A total of 13 participants (1.6\%) were excluded after randomization because they had veins greater than $15 \mathrm{~mm}$ in diameter (5 participants), recurrent varicose veins ( 3 participants), or coexisting conditions or absence of reflux (5 participants). Participants who were assigned to treatment with laser ablation or foam sclerotherapy had the option of retreatment with foam sclerotherapy for any residual varicosities at or after the 6-week follow-up assessment. One site performed concurrent phlebectomies at the time of laser therapy. 


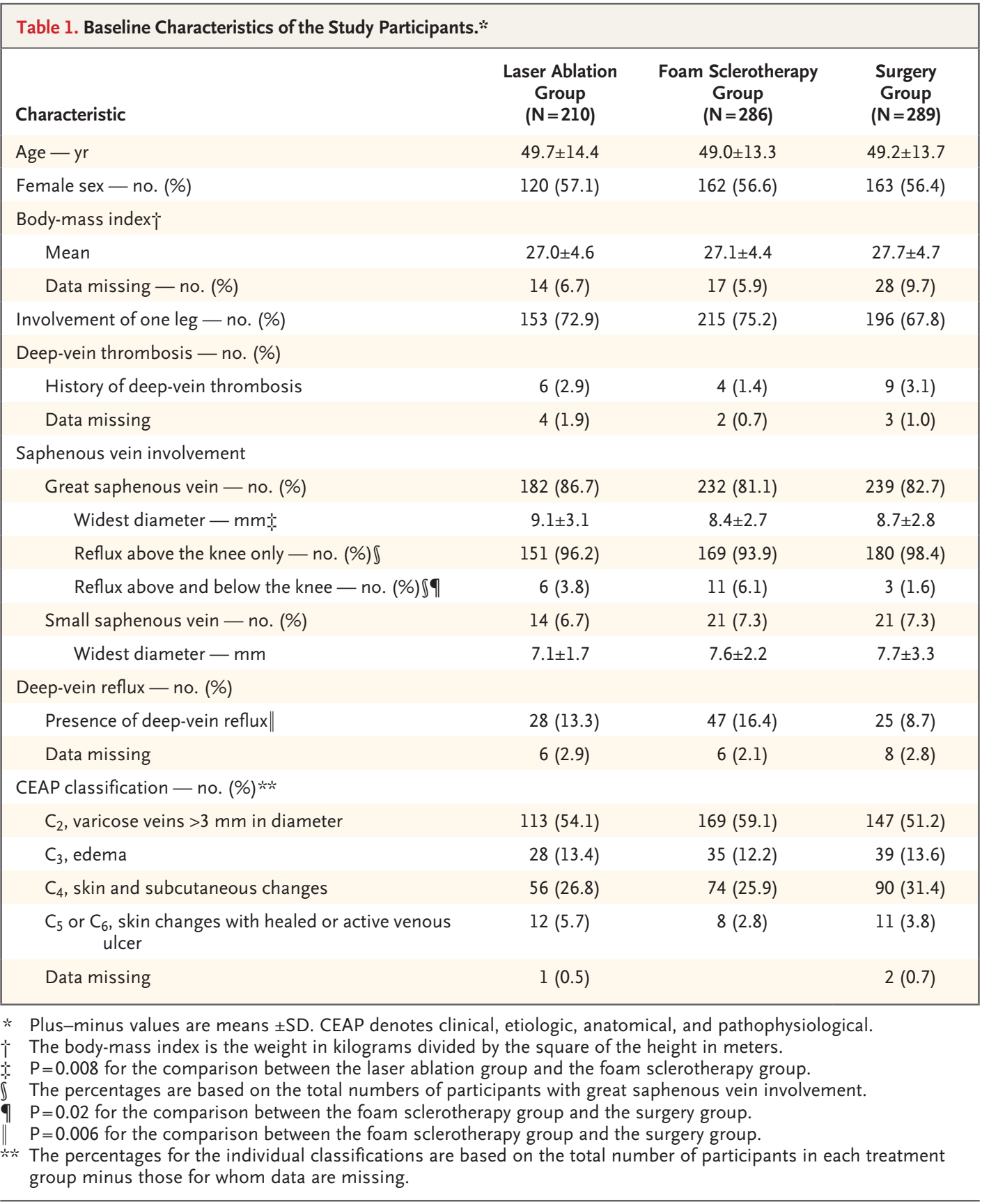

\section{SECONDARY OUTCOMES}

There were no major differences between groups in SF-36 subscales or EQ-5D VAS scores. With regard to patient satisfaction, the majority of participants were willing to repeat the same treatment and to recommend the treatment they had received to a friend (Tables S10 and S11 in the Supplementary Appendix).

At 5 years, $58 \%$ of the patients who had under- gone laser ablation, 54\% who had undergone surgery, and $47 \%$ who had undergone foam sclerotherapy reported having no varicose veins (odds ratio for foam sclerotherapy vs. laser ablation, $0.59 ; 95 \%$ CI, 0.41 to 0.85 ). Overall, $11 \%$ of the patients in the laser ablation group, 14\% in the foam sclerotherapy group, and $7 \%$ in the surgery group had further treatment.

The mean $( \pm S D)$ extent of varicose veins as 


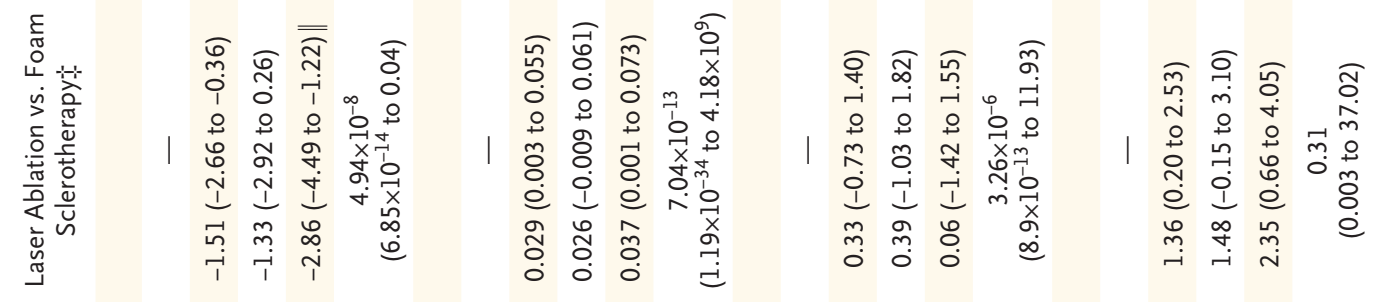

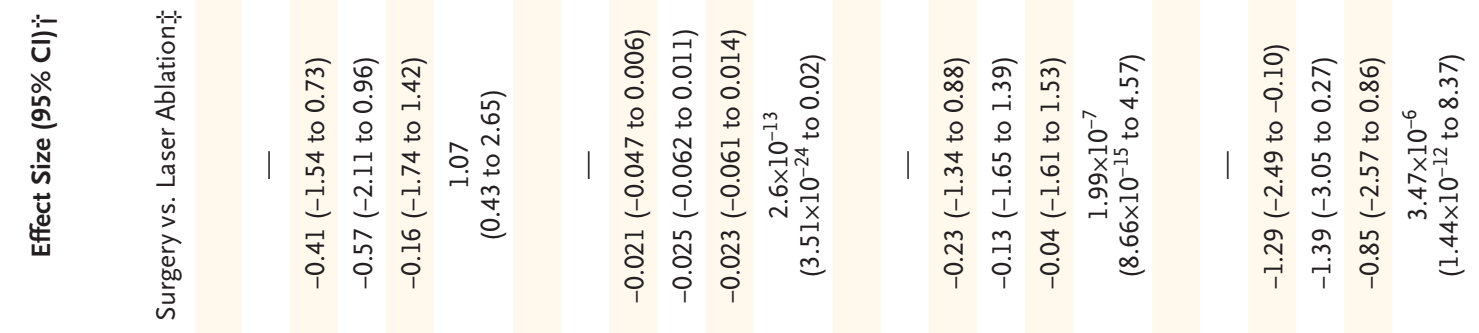

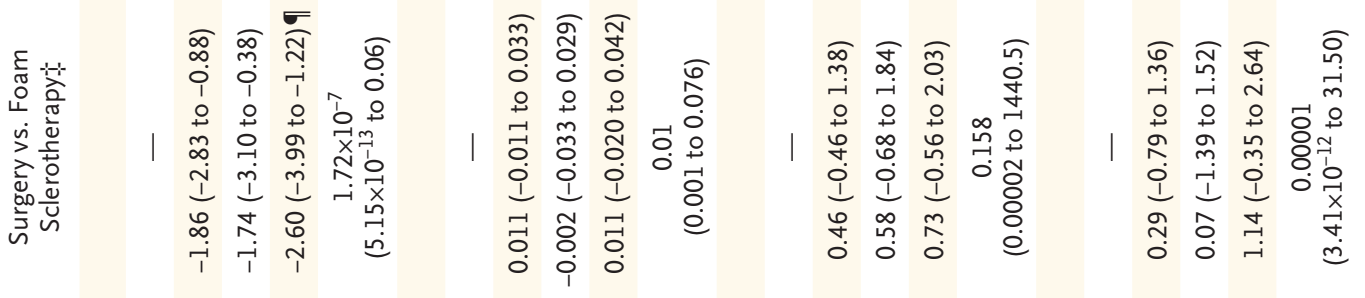

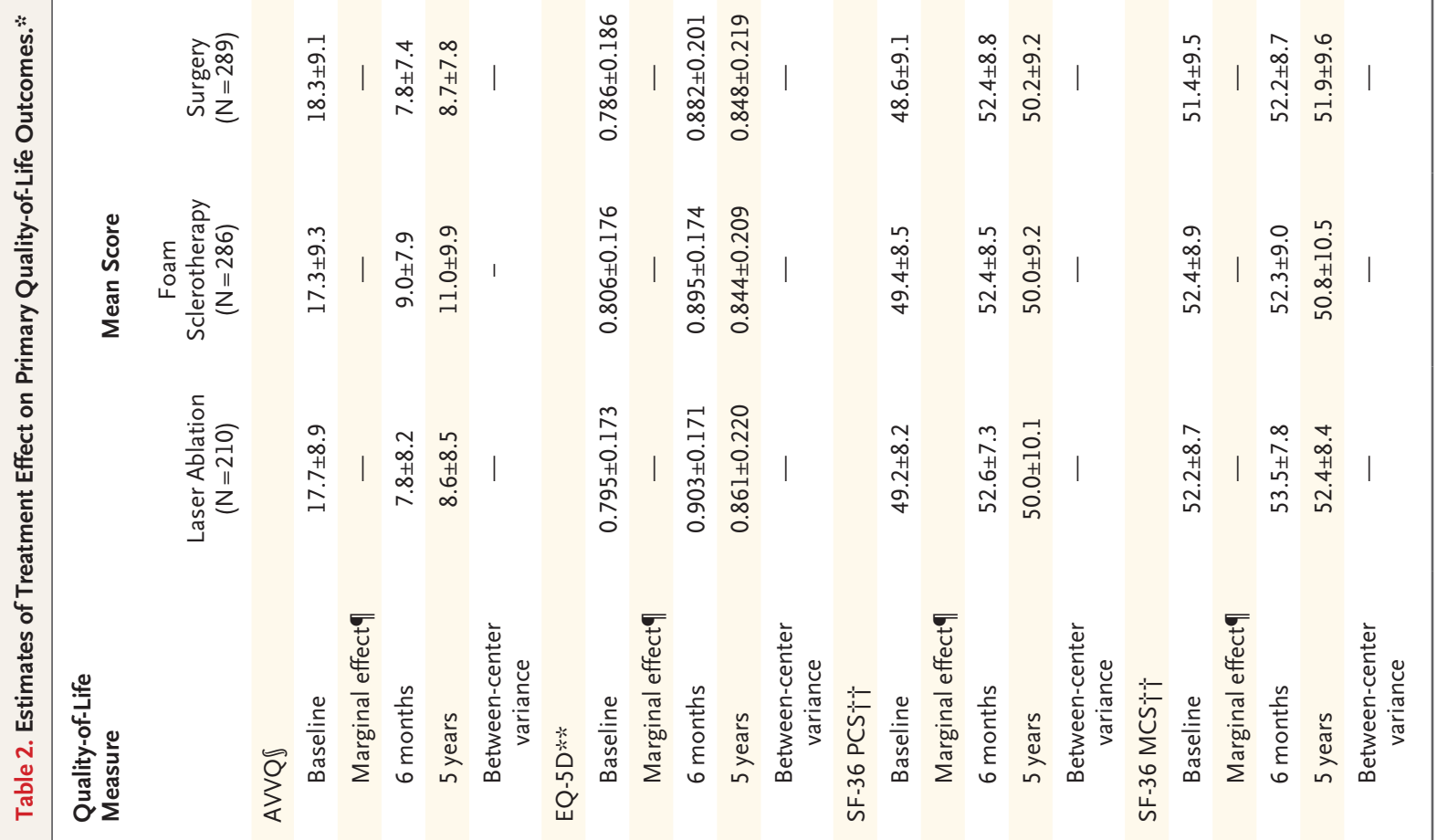




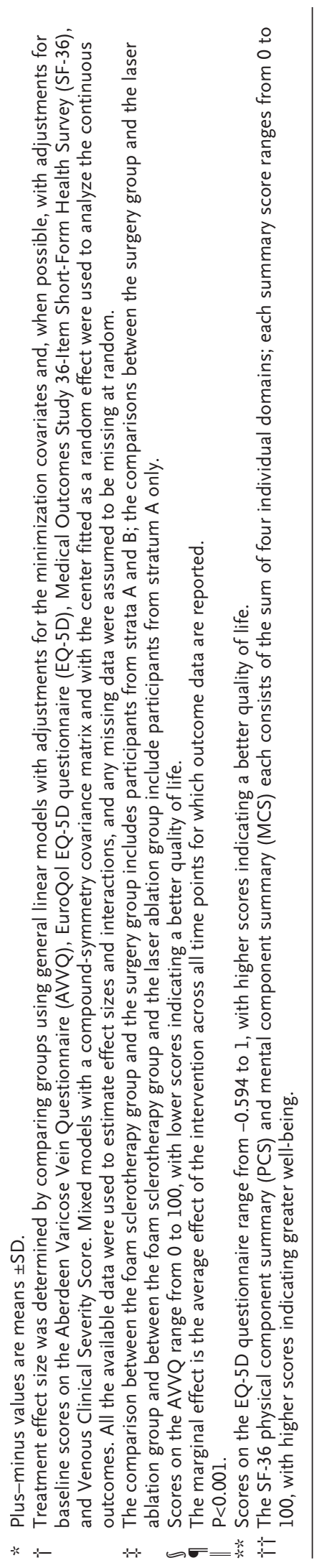

determined by the participant-reported VAS score was 2.6 \pm 2.2 with laser ablation, 3.2 \pm 2.5 with foam sclerotherapy, and $2.4 \pm 2.3$ with surgery (effect size for laser ablation vs. foam sclerotherapy, -0.79 ; $95 \% \mathrm{CI},-1.24$ to -0.34 ; and for surgery vs. foam sclerotherapy, $-0.74 ; 95 \%$ CI, -1.12 to -0.36 ). Similar scores were noted for the nurse-reported VAS scores. Venous Clinical Severity Score assessments were better at 5 years than at baseline in all three groups. The rates of complete success with respect to truncal-vein ablation at 5 years were $64.0 \%$ with laser ablation, $33.3 \%$ with foam sclerotherapy, and $75.9 \%$ with surgery. (Additional details on effect sizes are provided in Tables S12 and S13 in the Supplementary Appendix.)

The individual patient cost and utility data for the three-way analysis and two-way analysis, performed according to the intention-to-treat principle, are summarized in Tables S14 and S15, respectively, in the Supplementary Appendix. In the adjusted trial-data-based cost-effectiveness analysis, treatment with laser ablation had the highest chance $(71.6 \%)$ of being cost-effective in the three-way comparison, at a ceiling willingness-to-pay ratio of $£ 20,000(\$ 28,433)$ per QALY gained. In the two-way comparison, the incremental cost-effectiveness ratio for surgery as compared with foam sclerotherapy was below the willingness-to-pay threshold of $£ 20,000(\$ 28,433)$ per QALY gained in $83.3 \%$ of the bootstrapped samples (Table S16 in the Supplementary Appendix). There were no significant differences in QALYs among the treatment methods.

\section{DISCUSSION}

In this large, multicenter trial comparing surgery, laser ablation, and foam sclerotherapy for the treatment of primary varicose veins, quality of life was better at 5 years than at baseline in all groups. Five years after treatment, there were significant differences between the treatments with respect to disease-specific quality of life. The AVVQ scores were better among participants treated with laser ablation or surgery than among those treated with foam sclerotherapy, with effect sizes of 2.86 and 2.60, respectively. Quality of life was similar in the laser ablation and surgery groups. The 5-year cost-effective analyses were generally in keeping with our previously reported model-based estimates - name- 


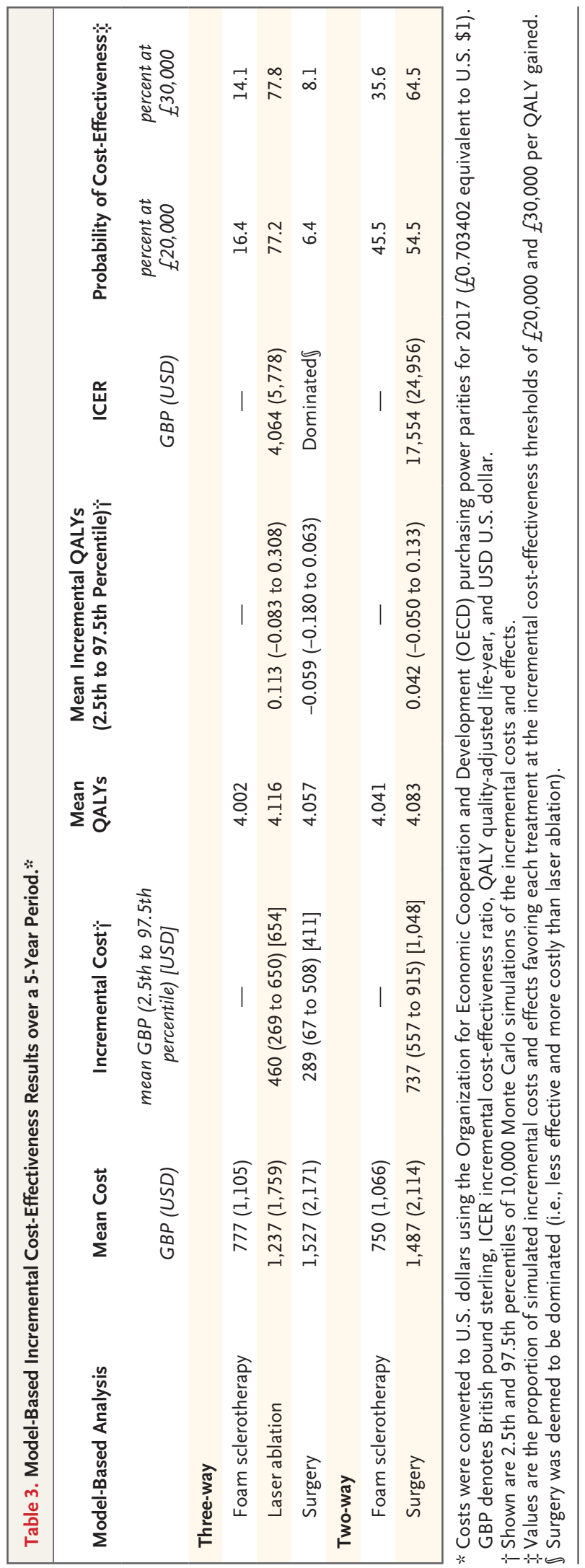

ly, laser ablation performed under a local anesthetic in a treatment-room setting had a $77 \%$ probability of being cost-effective up to a willingness-to-pay maximum of $£ 20,000(\$ 28,433)$. There was considerable uncertainty in the twoway cost-effective analysis comparing foam sclerotherapy with surgery.

One previous, smaller study showed that at 5 years after treatment there was greater improvement in disease-specific quality of life (a secondary outcome) among patients treated with laser ablation than among those treated with foam sclerotherapy, but no difference in diseasespecific quality of life was observed between patients who underwent foam sclerotherapy and those who underwent surgery. ${ }^{16}$ The differences in quality of life observed in our study are likely to relate to the fact that the presence and extent of varicose veins at 5 years were less in the laser ablation and surgery groups than in the foam sclerotherapy group, because disease-specific quality of life has previously been shown to be worse among patients who have recurrent disease than among those who do not. ${ }^{26}$ At 5 years, the presence of varicose veins after laser ablation as reported by the participants in this study was similar to the clinician-reported presence of varicose veins in two other studies ${ }^{14,15}$; in contrast, marginally fewer patients in this study who underwent surgery reported varicose veins at 5 years than the clinician-reported percentage (54\%) in the two other studies. ${ }^{14,15}$ The observed percentage of patients with successful truncal-vein ablation was higher among patients treated with laser ablation and surgery than among those treated with foam sclerotherapy, a finding that is consistent with results in other studies. ${ }^{16,27,28}$ It should be noted that patient satisfaction was high, which may partly explain the lower reintervention rates in this trial than in other trials. ${ }^{16,28}$ Whether restrictions on treatment for varicose veins within the National Health Service influenced retreatment rates is unclear.

The primary Markov model and the trial-databased analysis both showed laser ablation to be favored on the basis of cost-effectiveness. Despite a higher-than-predicted presence of varicose veins at 5 years $(42 \%$ after laser ablation, vs. $29 \%$ predicted; $46 \%$ after surgery, vs. $37 \%$ predicted; and $53 \%$ after foam sclerotherapy, vs. $40 \%$ predicted) and a lower reintervention rate, our results are generally in keeping with our 
previously reported model-based estimates derived from an existing network meta-analysis of trials. ${ }^{17,18}$ This previous meta-analysis included eight trials, the majority of which had less than 3 years of follow-up. ${ }^{29}$ Other Markov models have also shown laser ablation to have the highest chance of being cost-effective, ${ }^{30,31}$ whereas one has favored foam sclerotherapy. ${ }^{32}$ The modelbased two-way comparison indicated that surgery cost considerably more than foam sclerotherapy and generated a small, nonsignificant QALY gain. The magnitude of the QALY gain was sensitive to the analysis method, in that it was larger in the two-way trial-data-based analysis than in the three-way analysis, although it was still nonsignificant in the two-way analysis. The larger QALY gain in the two-way analysis as compared with the three-way analysis is partly explained by more unrelated deaths occurring in the foam sclerotherapy group during follow-up.

Limitations of our study include the lack of a sham procedure and the fact that patients and assessors were aware of the treatments. There was a substantial amount of missing data at 5 years, although the response rate with respect to participant-reported outcomes was higher than anticipated.

In summary, this large, multicenter trial comparing the clinical effectiveness of laser ablation, foam sclerotherapy, and surgery for the treatment of varicose veins showed that in all three groups, quality of life 5 years after treatment was improved from baseline. However, there were clinically important between-group differences in disease-specific quality of life that favored laser ablation and surgery over foam sclerotherapy. Laser ablation was similar to surgery with respect to quality of life and of the three treatments had the highest chance of being cost-effective.

The views and opinions expressed are those of the authors and not necessarily those of the National Institute for Health Research (NIHR) or the Department of Health and Social Care.

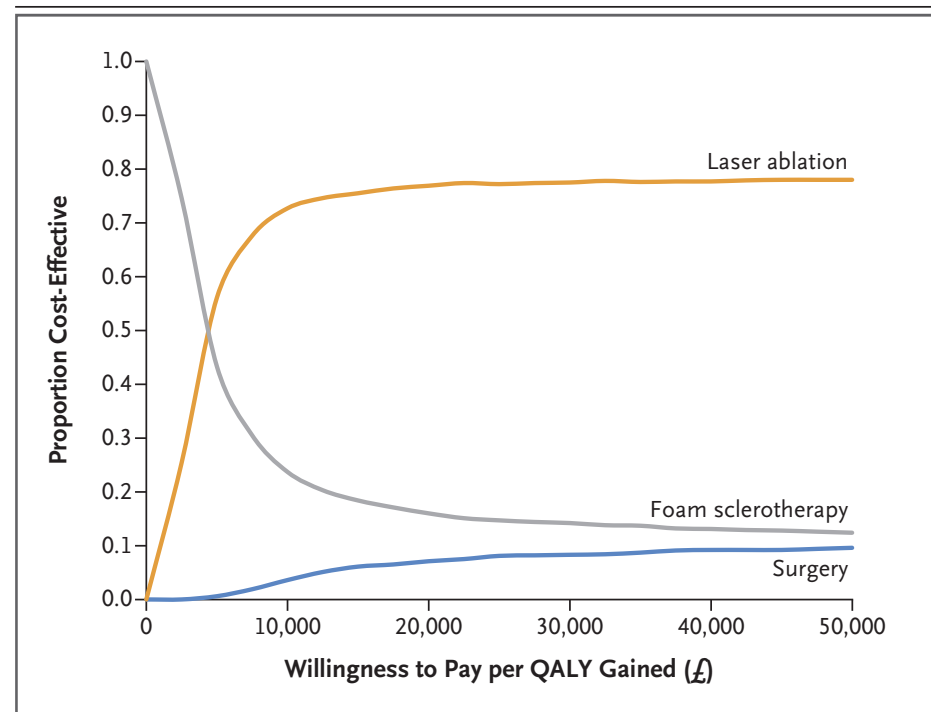

Figure 2. Markov-Model-Based Analysis of the Cost-Effectiveness of the Three Treatments at 5 Years.

Costs were converted to U.S. dollars with the use of the Organization for Economic Cooperation and Development (OECD) purchasing power parities for 2017 ( $£ 0.703402$ equivalent to $\$ 1$ ). With this conversion, the equivalent value for $£ 10,000$ is $\$ 14,217$; for $£ 20,000$ is $\$ 28,433$; for $£ 30,000$ is $\$ 42,650$; for $£ 40,000$ is $\$ 56,867$; and for $£ 50,000$ is $\$ 71,083$. More information about purchasing power parities is available at www.oecd.org/sdd/purchasing powerparitiespppsdata.htm.

A data sharing statement provided by the authors is available with the full text of this article at NEJM.org.

Supported by the NIHR Health Technology Assessment Program (Project number 06/45/02). The Health Services Research Unit is core-funded by the Chief Scientist Office of the Scottish Government Health and Social Care Directorate.

No potential conflict of interest relevant to this article was reported.

Disclosure forms provided by the authors are available with the full text of this article at NEJM.org.

We thank Janice Cruden for secretarial support and data management; Gladys McPherson, Mark Forrest, and the programming team at the Centre for Healthcare Randomized Trials; members of the Project Management Group for ongoing advice and support of the trial; the independent members of the trial steering committee and data and safety monitoring committee; and the staff at recruitment sites who facilitated recruitment, treatment, and follow-up of trial participants.

\section{APPENDIX}

The authors' affiliations are as follows: the Institute of Cardiovascular Research, University of Glasgow (J.B.), and the Institute of Applied Health Research, Nursing, Midwifery, and Allied Health Professions Research Unit, Glasgow Caledonian University (A.E.), Glasgow, the Health Services Research Unit (D.C., S.C.C., G.M., C.R.R., M.K.C.) and the Health Economics Research Unit (M.D., G.S., E.T.), University of Aberdeen, and the Department of Vascular Surgery, NHS Grampian, Aberdeen Royal Infirmary (P.B.), Aberdeen, the Edinburgh Clinical Trials Unit, University of Edinburgh, Edinburgh (J.N.), the School of Medicine, Medical and Biological Sciences, University of St. Andrews, St. Andrews (J.M.B.), the Department of Vascular Surgery, Royal Devon and Exeter Hospital, Exeter (B.C.), the Department of Vascular Surgery, Hull Royal Infirmary, Hull (I.C.), the School of Surgery, University of Leeds (M.G.), and Vascular Surgery, St. James University Hospital (J.S.), Leeds, Vascular Surgery, Gloucestershire Royal Hospital, Gloucester (J.E.), Vascular Surgery, Freeman Hospital, Newcastle upon Tyne (T.L.), the Vascular Surgical Unit, Royal Bournemouth Hospital, Bournemouth (S.A.B.), and the School of Health Sciences, City University of London, London (J.F.) - all in the United Kingdom. 
REFERENCES

1. Biemans AAM, Kockaert M, Akkersdijk $\mathrm{GP}$, et al. Comparing endovenous laser $\mathrm{ab}$ lation, foam sclerotherapy, and conventional surgery for great saphenous varicose veins. J Vasc Surg 2013;58(3):727-34.e1.

2. Samuel N, Carradice D, Wallace T, Mekako A, Hatfield J, Chetter I. Randomized clinical trial of endovenous laser ablation versus conventional surgery for small saphenous varicose veins. Ann Surg 2013;257:419-26.

3. Rass K, Frings N, Glowacki P, et al. Comparable effectiveness of endovenous laser ablation and high ligation with stripping of the great saphenous vein: two-year results of a randomized clinical trial (RELACS study). Arch Dermatol 2012; 148:49-58.

4. Carradice D, Mekako AI, Mazari FAK, Samuel N, Hatfield J, Chetter IC. Randomized clinical trial of endovenous laser ablation compared with conventional surgery for great saphenous varicose veins. Br J Surg 2011;98:501-10.

5. Rasmussen LH, Lawaetz M, Bjoern L, Vennits B, Blemings A, Eklof B. Randomized clinical trial comparing endovenous laser ablation, radiofrequency ablation, foam sclerotherapy and surgical stripping for great saphenous varicose veins. $\mathrm{Br} \mathrm{J}$ Surg 2011;98:1079-87.

6. Darwood RJ, Theivacumar N, Dellagrammaticas D, Mavor AI, Gough MJ. Randomized clinical trial comparing endovenous laser ablation with surgery for the treatment of primary great saphenous varicose veins. Br J Surg 2008;95:294-301. 7. Lattimer CR, Azzam M, Kalodiki E, Shawish E, Trueman P, Geroulakos G. Cost and effectiveness of laser with phlebectomies compared with foam sclerotherapy in superficial venous insufficiency: early results of a randomised controlled trial. Eur J Vasc Endovasc Surg 2012;43: 594-600.

8. Shadid N, Ceulen R, Nelemans P, et al. Randomized clinical trial of ultrasoundguided foam sclerotherapy versus surgery for the incompetent great saphenous vein. Br J Surg 2012;99:1062-70.

9. Brittenden J, Cotton SC, Elders A, et al. A randomized trial comparing treatments for varicose veins. N Engl J Med 2014;371: 1218-27.

10. Christenson JT, Gueddi S, Gemayel G, Bounameaux H. Prospective randomized trial comparing endovenous laser ablation and surgery for treatment of primary great saphenous varicose veins with a 2year follow-up. J Vasc Surg 2010;52:1234-41. 11. Rasmussen LH, Bjoern L, Lawaetz M, Lawaetz B, Blemings A, Eklöf B. Ran- domised clinical trial comparing endovenous laser ablation with stripping of the great saphenous vein: clinical outcome and recurrence after 2 years. Eur J Vasc Endovasc Surg 2010;39:630-5.

12. Rasmussen L, Lawaetz M, Serup J, et al. Randomized clinical trial comparing endovenous laser ablation, radiofrequency ablation, foam sclerotherapy, and surgical stripping for great saphenous varicose veins with 3-year follow-up. J Vasc Surg Venous Lymphat Disord 2013;1:349-56.

13. Gauw SA, Lawson JA, van Vlijmen-van Keulen CJ, Pronk P, Gaastra MT, Mooij MC. Five-year follow-up of a randomized, controlled trial comparing saphenofemoral ligation and stripping of the great saphenous vein with endovenous laser ablation (980 nm) using local tumescent anesthesia. J Vasc Surg 2016;63:420-8.

14. Rass K, Frings N, Glowacki P, Gräber $\mathrm{S}$, Tilgen $\mathrm{W}$, Vogt $\mathrm{T}$. Same site recurrence is more frequent after endovenous laser ablation compared with high ligation and stripping of the great saphenous vein 5 year results of a randomized clinical trial (RELACS). Eur J Vasc Endovasc Surg 2015;50:648-56.

15. Rasmussen L, Lawaetz M, Bjoern L, Blemings A, Eklof B. Randomized clinical trial comparing endovenous laser ablation and stripping of the great saphenous vein with clinical and duplex outcome after 5 years. J Vasc Surg 2013;58:421-6.

16. van der Velden SK, Biemans AA, De Maeseneer MG, et al. Five-year results of a randomized clinical trial of conventional surgery, endovenous laser ablation and ultrasound-guided foam sclerotherapy in patients with great saphenous varicose veins. Br J Surg 2015;102:1184-94.

17. Tassie E, Scotland G, Brittenden J, et al. Cost-effectiveness of ultrasound guided foam sclerotherapy, endovenous laser ablation or surgery as treatment for primary varicose veins from the randomized CLASS trial. Br J Surg 2014;101:1532-40.

18. Brittenden J, Cotton SC, Elders A, et al Clinical effectiveness and cost-effectiveness of foam sclerotherapy, endovenous laser ablation and surgery for varicose veins: results from the Comparison of LAser, Surgery and foam Sclerotherapy (CLASS) randomised controlled trial. Health Technol Assess 2015;19:1-342.

19. Garratt AM, Macdonald LM, Ruta DA, Russell IT, Buckingham JK, Krukowski $\mathrm{ZH}$. Towards measurement of outcome for patients with varicose veins. Qual Health Care 1993;2:5-10.

20. Smith JJ, Garratt AM, Guest M, Greenhalgh RM, Davies AH. Evaluating and improving health-related quality of life in patients with varicose veins. J Vasc Surg 1999;30:710-9.

21. Garratt AM, Ruta DA, Abdalla MI, Russell IT. Responsiveness of the SF-36 and a condition-specific measure of health for patients with varicose veins. Qual Life Res 1996;5:223-34.

22. Klem TM, Sybrandy JE, Wittens $\mathrm{CH}$, Essink Bot ML. Reliability and validity of the Dutch translated Aberdeen Varicose Vein Questionnaire. Eur J Vasc Endovasc Surg 2009;37:232-8.

23. EQ-5D. Rotterdam, the Netherlands: EuroQol (http://www.euroqol.org/).

24. SF-36 Health Survey update (http:// www.clintools.com/victims/resources/ assessment/health/sf36.html).

25. White IR, Horton NJ, Carpenter J, Pocock SJ. Strategy for intention to treat analysis in randomised trials with missing outcome data. BMJ 2011;342:d40.

26. Beresford T, Smith JJ, Brown L, Greenhalgh RM, Davies AH. A comparison of health-related quality of life of patients with primary and recurrent varicose veins. Phlebology 2003;18:35-7.

27. Vähäaho S, Halmesmäki K, Albäck A, Saarinen E, Venermo M. Five-year followup of a randomized clinical trial comparing open surgery, foam sclerotherapy and endovenous laser ablation for great saphenous varicose veins. Br J Surg 2018;105: 686-91.

28. Lawaetz M, Serup J, Lawaetz B, et al. Comparison of endovenous ablation techniques, foam sclerotherapy and surgical stripping for great saphenous varicose veins: extended 5-year follow-up of a RCT. Int Angiol 2017;36:281-8.

29. Varicose veins: diagnosis and management - clinical guideline CG168. London: National Institute for Health and Care Excellence, July 2013 (http://guidance .nice.org.uk/CG168).

30. Marsden G, Perry M, Bradbury A, et al. A cost-effectiveness analysis of surgery, endothermal ablation, ultrasound-guided foam sclerotherapy and compression stockings for symptomatic varicose veins. Eur J Vasc Endovasc Surg 2015;50:794-801.

31. Gohel MS, Epstein DM, Davies AH. Cost-effectiveness of traditional and endovenous treatments for varicose veins. $\mathrm{Br} \mathrm{J}$ Surg 2010;97:1815-23.

32. Carroll C, Hummel S, Leaviss J, et al. Clinical effectiveness and cost-effectiveness of minimally invasive techniques to manage varicose veins: a systematic review and economic evaluation. Health Technol Assess 2013;17:1-141.

Copyright (c) 2019 Massachusetts Medical Society. 